\title{
Method for Constructing the Topological Structure of an Access Network with a Distributed Structure
}

\author{
Nishanbayev Tuygun Nishanbayevich, Abdullaev Mirjamol Mirkamilovich, \\ Matkurbonov Dilshod Matkurbon Ogli \\ Department of Data Communication Networks and System, Telecommunication Faculty, Tashkent University of Information Technologies \\ Named After Muhammad al-Khwarizmi, Tashkent, Uzbekistan
}

\section{Email address:}

dilshod91_93dn@mail.ru(M. D. M. Ogli)

\section{To cite this article:}

Nishanbayev Tuygun Nishanbayevich, Abdullaev Mirjamol Mirkamilovich, Matkurbonov Dilshod Matkurbon Ogli. Method for Constructing the Topological Structure of an Access Network with a Distributed Structure. Advances in Networks. Vol. 6, No. 1, 2018 , pp. 14-20. doi: $10.11648 /$ j.net.20180601.12

Received: February 12, 2018; Accepted: March 5, 2018; Published: March 26, 2018

\begin{abstract}
The article proposes a method for constructing the topological structure of the access network of the information and communication network (ICN), which has a distributed structure. The essence of the method is reduced to the fact that initially the region is divided into zones, then in each zone a star-shaped graph with a vertex in one of the possible locations of the switch is formed. When forming the subnet structure in the next zone, a fixed set of terminal systems adds the point where the switch of the previous zone is located. This approach allows, in the construction of a subnet topology in zones, to simultaneously form one connected (core) topology of the backbone network between the zones. In the next stage, the topology of the desired K-connected structure of the distributed access network of the ICN is constructed. The problem is solved by using sections of dominant sets and P-medians of graph theory.
\end{abstract}

Keywords: Access Network, Optical Networks, Info-Communication Network, Backbone Network, Graph Theory, Orthogonal Metric, Distributed Network Structure, Terminal Equipment

\section{Introduction}

The topological structure of the access level of the infocommunication network (ICN) at the current stage of the development of network technologies is a complex distributed structure and consists of broadband communication channels and the corresponding broadband network equipment. The access network can be built only from optical lines, but with a gradual transition from cable network to optical one, it can have a hybrid structure, communication channels at different sites have different capacity. In general, the ICN access network consists of terminal equipment, switching devices and communication channels, designing and creating it is a timeconsuming process that requires considerable capital investment $[1,2,3]$.

The access network by connecting the global infocommunication network to the backbone transport network provides access to the dislocated information and other resources of the ICN, allows for the exchange of data between dispersed subscribers distant from each other over long distances [8, 9, 10]. It also provides interworking between subscribers located within the considered access level of ICN.

If the access network covers a sufficiently large territory, then the terminal equipment will be located in different parts of the region in question and the task arises of constructing a topological structure of the access network that has access to the backbone network. Optimal placement of optical switches and their connection with each other and with terminal means will significantly reduce the cost of their creation $[6,7]$.

\section{The Method of Forming the Topological Structure of the Access Network}

The essence of the method is to determine the number of switching equipment (optical switches and routers), the coordinates of their installation, the ways of connecting them 
with terminal equipment, and with each other and with the network equipment of transport level ICN. The access network (AN) has a rather complex distributed structure and the construction of its topology is one of the main tasks of the design of the ICN. This task includes the determination of the number and location of switches, the optimal choice of connection scheme for terminal equipment with switches, the construction of a scheme for connecting distributed.

The method allows sequentially determining the structure of terminal subnets in parallel to form a single-connected (core) topology of the communication subnet and purposefully construct the topology of the K-related structure of the desired access network (the parameter $\mathrm{K}$ characterizes the number of connections between the access network switches, $\mathrm{K}=1,2,3, \ldots \mathrm{M}-1, \mathrm{M}$ is the number of switches in AN).

The construction of the access network topology is carried out in two stages: in the first phase, the topology of the terminal subnet and the core network of the communication network are constructed, and in the second one, a $\mathrm{K}$ connected network of the communication access network is built.

The solution to this problem begins with partitioning the region into separate zones, each of which should be serviced as far as possible by means of its own subnet. At the same time, the following are considered given:

The territorial and administrative parameters of the region, where it is planned to create an access network with a distributed structure;

Coordinates of placement of terminal equipment; the coordinates of the possible locations of switches, etc.

In each zone, the number of terminal equipment is determined (taking into account the increase in their number in the process of network development). The division of the region into service areas is made according to the degree of compliance of the optical switch's power.

Formation of the topology of the terminal subnet in the zone is realized as follows.

Concentrated in the zones of terminal equipment are served by one switch, so the initial topology is a set of starshaped structures (graphs) with centers at one of the possible locations of the switch.

The optimal structure is the minimum sum of channel weights-rays. The center will be the desired point of the optimal location of the switch. Synthesis in the next zone is implemented in a similar way, but a fixed set of terminal systems adds one point where the switch of the previous zone is located. The choice of the location of the switch in the next zone depends on the number and location of the switch in the previous zone.

Since at this stage only one connection is organized between neighboring zones, the variant with the least weight is naturally chosen. In such a formulation, a matter of essential importance is the sequence of selection of zones for synthesis.

The principle of successive transition to synthesis under the networks of the corresponding zones is as follows.
Each switch of the analyzed zone must connect to one of the possible switching points of the switch of the next zone, provided that the switch of this zone does not have a connection with the switches of other zones.

If the solution of the synthesis problem in the next zone led to the establishment of a connection between its switches and the switch of the previous zone, but when building a network in subsequent zones, a zone with a switch with a lower weight is identified, then the previously established connection is broken and a connection is established with the switch of this zone.

As a result of viewing all zones, an interconnected structure of star graphs is constructed, the centers (vertices) of which are connected by shortest paths. In other words, the shortest single connected graph is constructed, which is the basis for constructing a $K$-connected structure with the required properties.

\subsection{Fundamentals of the Theory of Dominant Sets and P- Medians of a Graph}

This problem can be solved by combinatorial methods using sections of dominant sets and the P-median of graph theory $[4,5]$.

As shown in the graph theory, for the graph $G=(X, D)$ (where $\mathrm{X}$ is the set of vertices, $\mathrm{D}$ are the correspondences that show how the vertices are connected to each other and determines the end vertices of the arcs for which the initial vertex is the subset $X_{1}, X_{2}, \ldots$, is the vertex $X_{i} \in X$ ) is the set of vertices $S \in X$ chosen so that for each vertex $X_{j}$ not in $S$ there corresponds an arc from some vertex of $S$ to the vertex $\mathrm{X}_{\mathrm{j}}$, that is.

$$
S \cup D(S)=X_{j}
$$

Since there is a set of minimal dominant sets, then if $\mathrm{P}$ is the family of all minimal dominant subsets of the graph, then the number.

$$
\begin{gathered}
\beta[G]=\min |S| \\
\mathrm{S} \in \mathrm{P}
\end{gathered}
$$

is the dominance number of the graph $\mathrm{G}$, and the set $\mathrm{S}$ on which the minimum is attained is the smallest dominant set (SDS).

In other words, SDS is the smallest number of vertices from which all other vertices of the graph are accessible. Directly from the definition of SDS, the notion of the median of a graph is derived.

For each tops $\mathrm{X}_{\mathrm{I}} \in \mathrm{X}$, two gear ratios are defined

$$
\begin{aligned}
& \delta_{\mathrm{o}}\left(x_{i}\right)=\sum_{x_{j} \in M} V_{j} * d\left(X_{i}, X_{j}\right), \\
& \delta_{t}\left(x_{i}\right)=\sum_{x_{j} \in M} V_{j} * d\left(X_{j}, X_{i}\right),
\end{aligned}
$$

where $d\left(X_{i}, X_{j}\right)$ is the shortest distance from the vertex $X_{i}$ to the vertex $X_{j}$;

$\mathrm{V}_{\mathrm{j}}$ is the weight of the corresponding vertex; 
$\delta_{o}, \delta_{\mathrm{t}}$ are, respectively, the outer and inner gear numbers of the vertex $X_{i}$ and are the sums of the elements of the row of the matrix obtained by multiplying the column of the distance matrix $D(G)=\left[d\left(x_{i}, x_{j}\right)\right]$ by the weight corresponding to this column of the vertex $\mathrm{v}_{\mathrm{j}}$;

The vertices $\overline{\mathrm{x}}_{o}$ and $\overline{\mathrm{x}}_{t}$ for which

$$
\begin{gathered}
\delta_{0}\left(\overline{\mathrm{x}}_{\mathrm{o}}\right)=\underset{\mathrm{x}_{\mathrm{i}} \in \mathrm{X}}{\min \left[\delta_{\mathrm{o}}\left(\mathrm{x}_{\mathrm{i}}\right)\right]}, \\
\delta_{t}\left(\overline{\mathrm{x}}_{t}\right)=\underset{x_{i} \in \mathrm{X}}{\min \left[\delta_{\mathrm{t}}\left(\mathrm{x}_{\mathrm{i}}\right)\right]},
\end{gathered}
$$

are the outer and inner medians of $\mathrm{G}$.

Thus, if $\mathrm{S}$ contains $\mathrm{P}$ vertices and is a subset of the set of vertices of $X$, then $P$ is the median of the graph $G$ is a subset of P-points from which all the remaining points of the set $\mathrm{X}$ are attained for the minimal sum of distances from them and the problem of finding the P- central in the general class of problems of distribution and location of service centers, to which the task of optimal placement of the switch can be directly related. In other words, the median vertex of a graph is a vertex, the sum of the distances from which to other vertices is minimal.

\subsection{The Method of Forming the Access Network Topology on the Basis of the Theory of Constructing a P-Median of a Graph}

It should be pointed out that all that has been said concerning oriented graphs extends to undirected graphs. In this case, the indices a and $t$ in the corresponding equations will be absent and the equation for the P-median is reduced to the following expression.

$$
\delta\left(x_{p}\right)=\sum_{x_{j} \in M} V\left(X_{p}, X_{j}\right),
$$

For non-oriented graphs, the concepts of external and internal medians are meaningless, since their values are equal to each other. Proceeding from this, the problem of determining the P-median for an undirected graph can be expressed in the terminology of the solved problem of determining the topology of the access network.

To do this, let's imagine the set of all the points of location of the terminal equipment (TE), as well as all possible locations for the switches, as a common set of vertices of $\mathrm{X}$ of some non-oriented graph $\mathrm{G}$ with a star like structure.

Since the access network is a distributed structure consisting of M-service areas and, hence, from M-thermal subnets, each of these networks is described by a star-shaped graph $\mathrm{G}_{\mathrm{m}}$ with the set of vertices $\mathrm{X}^{\mathrm{m}}$.

Let $X_{q}^{m}$ - the set of vertices $X^{m}$ of the graph $G_{m}$, where $q$ is the number of possible locations for the switches in the m-th subscriber network. Consequently, the gear ratio of the graph $\mathrm{G}_{\mathrm{m}}$ will be equal to

$$
\delta^{m}\left(X_{p}^{m}\right)=\sum_{X_{j} \in X^{m}} V_{j} d\left(X_{p}^{m}, j\right)
$$

and the set $\bar{X}_{\mathrm{p}}^{\mathrm{m}}$ for which

$$
\begin{gathered}
\delta^{\mathrm{m}}\left(\overline{\mathrm{X}}_{\mathrm{p}}^{\mathrm{m}}\right)=\min \left[\delta^{\mathrm{m}}\left(\mathrm{X}_{\mathrm{p}}^{\mathrm{m}}, \mathrm{j}\right)\right], \\
\mathrm{X}_{\mathrm{p}}^{\mathrm{m}} \in \mathrm{X}^{\mathrm{m}}
\end{gathered}
$$

is the P-median of the graph $\mathrm{G}_{\mathrm{m}}$, which corresponds to the required points of the optimal placement of the switch in the $\mathrm{m}$ - th zone of AN. Since the zone is a star-shaped structure with one or two vertices in which the switches are placed, the problem of finding the P-median reduces to the cases of 1 or 2 medians. The task of searching in terms of integer programming is formed as follows.

The task of searching in terms of integer programming is formed as follows.

Let the distribution matrix [ $\left.\xi_{\mathrm{ij}}\right]$ be given, in which

$$
\xi_{\mathrm{ij}}=\left\{\begin{array}{c}
1 \text { if } \text { he vertex } \mathrm{X}_{\mathrm{j}} \text { is attached to the vertex } \mathrm{X}_{\mathrm{i}} \\
0, \text { otherwise, }
\end{array}\right.
$$

$\xi_{\mathrm{ij}}=1$ in the case where $X_{i}$ is the median vertex and $\xi_{\mathrm{ij}}=0$ otherwise.

The problem of determining the P-median reduces to minimizing the function

$$
Z^{m}=\sum_{i=1}^{q} \sum_{j=1}^{n} d_{i j} \xi_{i j}
$$

where $-n$ is the total number of vertices of the graph $G_{m}$, i.e. the total number of terms of the set $\mathrm{X}^{\mathrm{m}}$ under the following restrictions:

a) any given vertex $X_{j}$ is attached to one and only to one median vertex $X_{i}$, i.e.

$$
\sum_{i=1}^{q} \xi_{i j}=1 \text { for } j=\overline{1, n}
$$

b) the existence of exactly $\mathrm{P}$ - median vertices is guaranteed

$$
\sum \xi_{i j}=P
$$

c) the attachment is made only to the vertices of the median set

$$
\xi_{\text {ij }} \leq \xi_{\text {ii }} \text { for all } \mathrm{i}=\overline{1, \mathrm{q}, \mathrm{J}=\overline{1, \mathrm{n}} .}
$$

When solving the problem of synthesis by this method, the key is to determine the weight characteristics of the edges of the graph $G_{m}$, which are directly related to the parametric characteristics of the external traffic entering the access terminal equipment, throughput and the technical capabilities of the switch.

The incoming information flows from the TE to the communication subnet are transmitted to their destinations via the real channels of communication, which have a finite capacity, and the higher the intensity of these streams, the 
higher their throughput capacity should be. If the capabilities of the dedicated communication channel do not satisfy the condition of reliability of the stream, then it is necessary to increase the capacity of the communication channel.

Note that under the capacity of the communication channel is understood not technical speed of information transmission, but information transmission speed, taking into account the necessary means of protecting information from errors, providing the specified fidelity.

A similar situation is observed in the organization of data exchange between switches. So, the cost of renting the channel $\mathrm{C}_{\mathrm{ij}}$ depends both on the distance $\mathrm{L}_{\mathrm{ij}}$, i.e. from the tariff $\mathrm{t}\left(\mathrm{L}_{\mathrm{ij}}\right)$, and on the informational speed of the given communication channel between $i-j$ switches $R_{i j}$ and is a functional

$$
\mathrm{C}_{\mathrm{ij}}=\Phi[\mathrm{t}(\mathrm{L}), \mathrm{Rij}]
$$

where $C_{i j}$ - the cost of renting the channel between the $i-j$ switches of the network.

Thus, when constructing a topology as the weights of the edges (communication channels), the choice of the parameter characterizing the cost of leasing the communication channels is justified, since the technical, informational and economic indicators of the access network are associated with it.

When choosing the weight of the ribs, one must take into account the obligatory fulfillment of the condition on the realizability of the flow. This indicates that the calculated intensity of the ordering of applications in the channel between the $\mathrm{i}-\mathrm{j}$ switches $\mu_{\mathrm{ij}}$ on the basis of the information speed of the given channel must correspond to the intensity of the external traffic $\gamma$ entering the given TE.

The expression $v_{j}^{m}$, representing the weight of the jth vertex of the star-shaped graph of the $\mathrm{m}$-zone, in this case will reflect the flow characteristics of the transport network. When all situations are taken into account, expression (1) for determining the $\mathrm{P}$-median for the case $\mathrm{P}=1$ in the most general form will have the form

$$
\mathrm{Z}^{\mathrm{m}}=\sum_{\mathrm{i}=1}^{\mathrm{q}} \sum_{\substack{\mathrm{j}=1 \\ \mathrm{j} \neq 1}}^{\mathrm{n}}\left\{v_{\mathrm{i}}^{\mathrm{m}}\left(\gamma, \mu_{\mathrm{ij}}\right) * \mathrm{C}_{\mathrm{ij}}^{\mathrm{m}}\left[\mathrm{t}(\mathrm{L}), \mathrm{R}_{\mathrm{ij}}\right] * \xi_{\mathrm{ij}}\right\},
$$

$v_{i}^{m}\left(\gamma, \mu_{i j}\right)$ - characterizes the flow characteristics of the transport network;

$\mathrm{C}_{\mathrm{ij}}^{\mathrm{m}}\left[\mathrm{t}(\mathrm{L}), \mathrm{R}_{\mathrm{ij}}\right]$ - characterizes the cost of leasing the communication channel between the node $i$ and the median "point" $\mathrm{j} ; \xi_{\mathrm{ij}}=1$, if $\mathrm{j}$ is the median point, otherwise $\xi_{\mathrm{ij}}=0$.

However, the described characteristics of communication channels cannot be considered final, since it does not take into account the part of the information streams coming to this channel from the remaining zones of the network.

This cannot be taken into account at this stage of the synthesis, because of the uncertainty of the a priori intensity of this part of the flow. Such corrections in all zones are realized when constructing a finite version of a graph with a given connectedness and solving the problem of the distribution of flows.
Thus, the initial topology in the zone is a star-shaped structure centered at one of the possible locations of the switch. The optimal structure is the minimum sum of channel weights-rays. The center will be the desired point for the optimal placement of the switch.

Synthesis in the next zone is implemented similarly, but a fixed set of terminal equipment adds one point where the switch of the previous zone is located. The choice of the location of the switch also depends on the number and location of the switch in the previous zone.

Since at this stage only one connection is organized between neighboring zones, the variant with the least weight is naturally chosen. With such a formulation, the question of essential importance is the sequence of selection of zones for synthesis.

The principle of successive transition to the synthesis of subnets of the corresponding zones is as follows.

Each switch of the analyzed zone is connected by one of the possible switching points of the switches of the next zone, provided that the switch of this zone has no connection with the switches of other zones.

If the solution of the synthesis problem in the next zone led to the establishment of a connection between its switches and switches of the previous zone, but when the network is built in subsequent zones, a zone with a switch with a lower weight is detected, then the previously established connection is broken and a connection is established with the switch of this zone.

Since each possible switch placement point (PSPP) has its coordinates $\left(\mathrm{x}_{\mathrm{i}}, \mathrm{y}_{\mathrm{i}}\right)$, then the parameter space defining its position in this zone must be metric.

The metric of a given set is the distance between specific pairs of points, i.e.

$$
P=\left(\left|\mathrm{x}_{i}-\mathrm{x}_{j}\right|+\left|y_{i}-y_{j}\right|\right)
$$

and the search is carried out in the metric space of parameters with the metric $\mathrm{P}$.

\subsection{Algorithm for Solving the Problem of Building an Access Network Topology}

The operation of the algorithm is represented by the sequence of the following procedures:

1. Using the orthogonal metric for all matching of the PSPP points in the considered zone, the value of $L(i, j)$ is calculated - the distance between pairs of points taking into account their coordinates describing the mutual position within the serviced zone.

2. The q-variant of the pair of points of the chosen zone is chosen in a random way and the star-shaped graphs are constructed, after which the value of the total weight of the edges $\mathrm{C}_{q}$

$$
C_{q}=\sum_{a=1}^{(n+k)_{2}} C_{a}+C_{1,2}
$$

3. The minimum set is chosen from the obtained set $\mathrm{C}_{q}$ and 
it is taken as the desired center and the transition to the search for the location of the switch in the next zone is carried out.

4. All terminal and hanging vertices of the spanning graph are defined.

5. The connection of all terminal and pendant vertices is made on the basis of the algorithm for constructing the minimum weight cycle and the associated structure of the access network is formed. On the generated topological structure, routing tasks are solved between the access network switches.

\section{Implementation of the Algorithm and Analysis of the Results}

To assess the effectiveness of the proposed method for constructing the topology of the access network, a computational experiment was carried out, for this purpose a program was developed in the language of JAVA, which performs the following functions:

Separates the given region into zones. The coordinates of the network elements of each zone in dynamic mode are entered in the computer memory. On one side of the monitor, the coordinates of the network elements are placed. For clarity, the figure shows an example for the simplest case.

Defines in each zone from among the given "points" the optimal "point" and its coordinates, where the switch will be installed and connects the network elements of the zone with it;

Takes the best solution to connect switches each zone to each other - defines the hanging and end vertices, which are connected with a lying near the top. The number of connections between switches must be at least two.

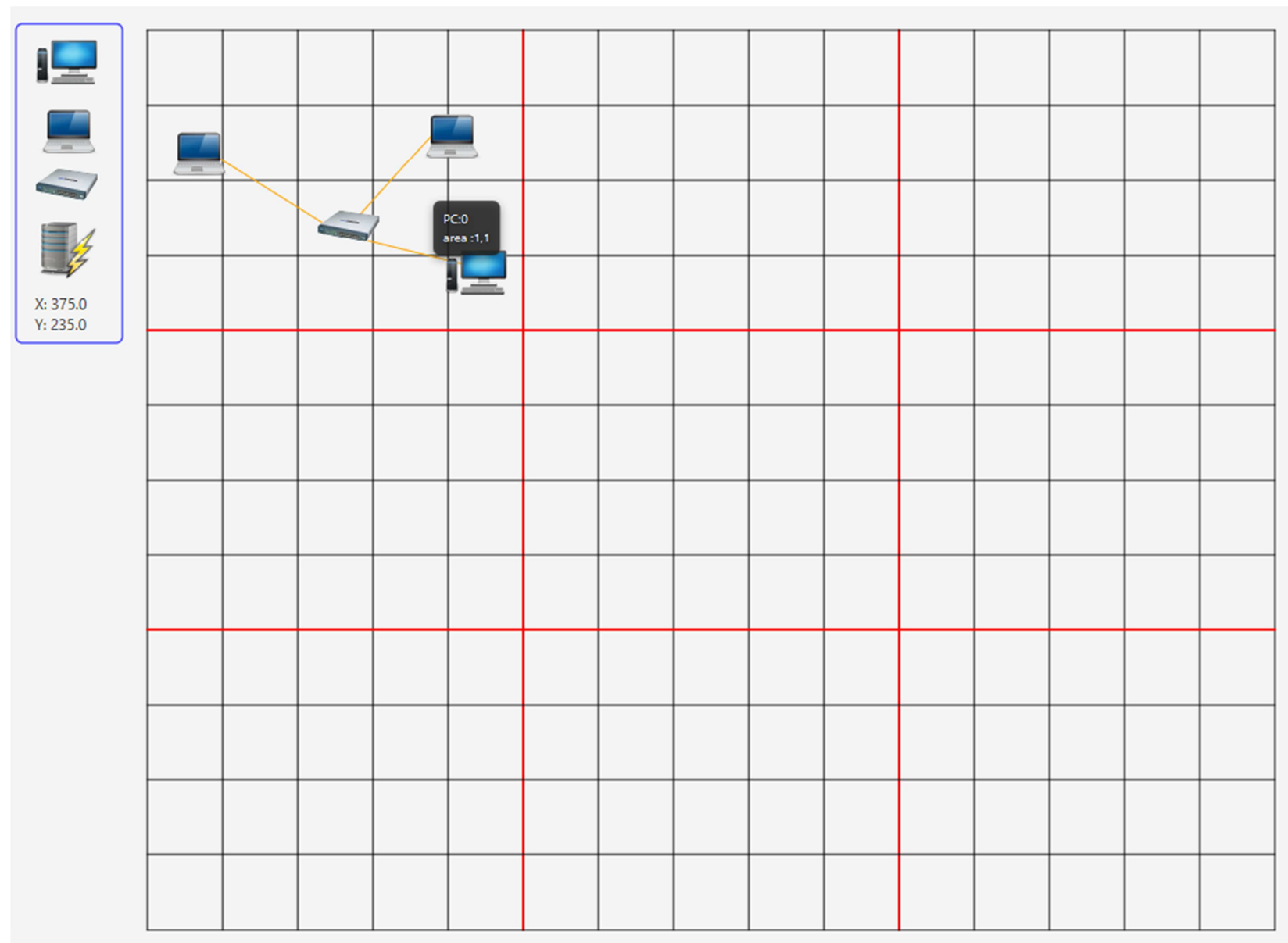




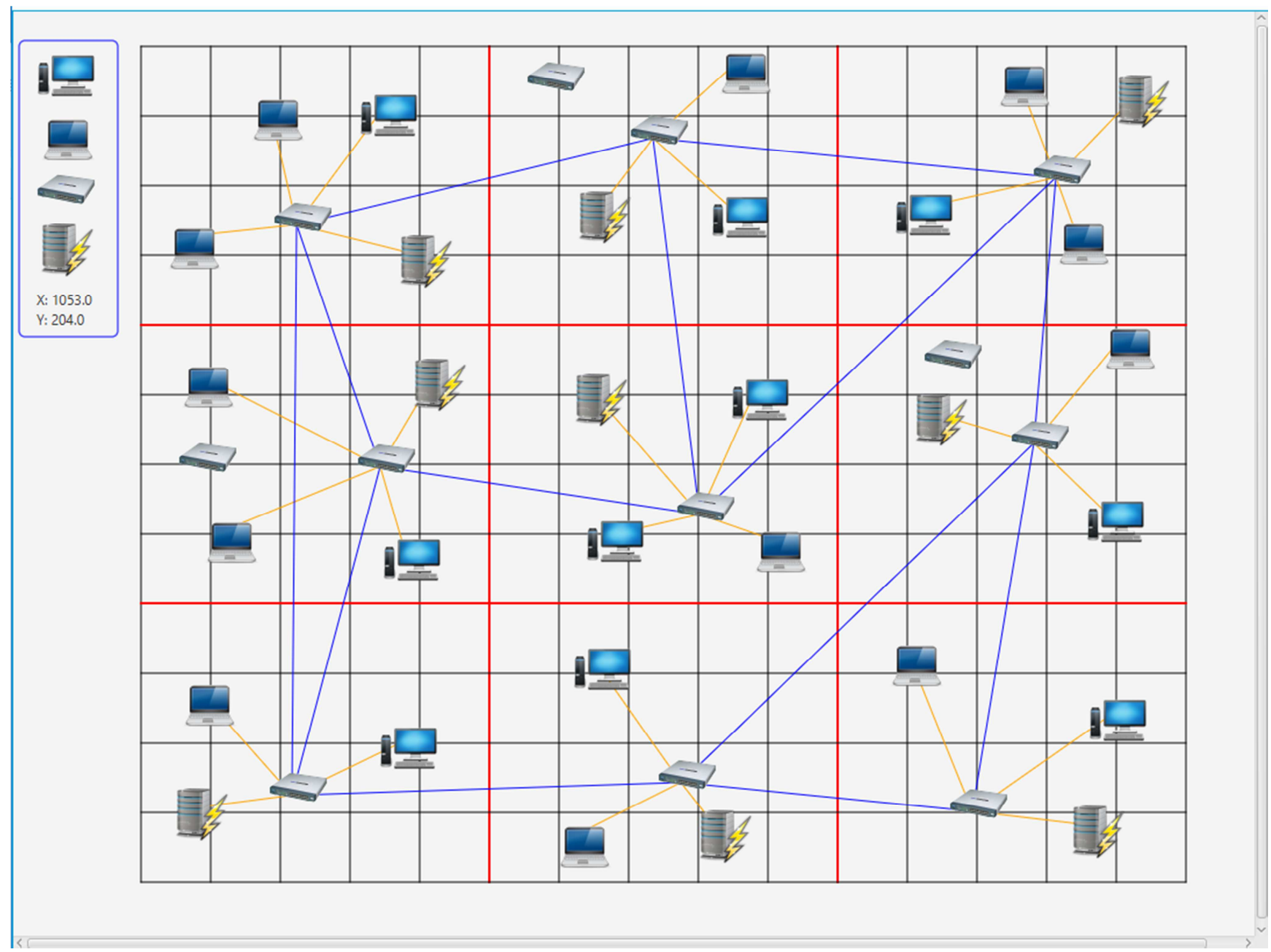

Figure 1. Example of the formation of the access network topology in the JAVA language.

A computational experiment was carried out for the region, consisting of 16 zones. Each zone was asked the coordinates of the terminal systems and the potential location of the switch. The results obtained by the proposed method compared with the method of the Autonomous formation of network topology without taking into account the location of switches in the next area. Analysis of the results showed that the proposed method has allowed to reduce expenses by 8 $10 \%$ in comparison with the method of construction of the access network without considering the characteristics of adjacent zones.

A program called "network Structure" sent for registration to the Agency for intellectual property of Uzbekistan.

\section{Conclusion}

At the present stage of development of IT technology choice of the optimal structure of the network topology is one of the main objectives in the design and creation of infocommunication systems and networks.

It should be noted that in some cases when building large corporate info-communication networks with distributed structure the topological structure of the desired network is considered given, i.e. it is based on the structure of the primary network context, and taking into account its characteristics are determined by the location of network equipment, it connects to the terminal assets and computer equipment that performs the functions of processing and storage. To connect them together uses the communication channels of the existing network of public communication. Further, if necessary, thus created a transport network is built up by adding network and communication equipment.

Created in this "primitive" way of the telecommunication network does not always lead to desired results - some part of the network will have a singly linked structure and to increase the number of links is not possible, and the other has multiple ties with neighboring switches. This eventually lead to the fact that in one area the network is heavily loaded, the other is underutilized, etc in such a network, as a rule, not met quality requirements QoS $[11,12]$.

It is therefore advisable to build the topological structure of OCOG purposefully considering the situation, not just "today" and "tomorrow" day. The proposed method of formation of the network topology will allow us to generate network topology while taking into account the random factors that may arise in the operation of the network. 


\section{References}

[1] Nikulsky I. E. Optical interfaces of digital switching stations and access networks. M .: Technosphere, 2006.

[2] Sokolov N. A. Subscriber Access Networks. Principles of construction. // ZAO "IG" "Enter Profi", 1999.

[3] Goldstein B. S., Kucheryavy A. E. Post-NGN communication networks SPb: BHV-Petersburg, 2013. - 160 p.

[4] M. Swami, K. Thulasiraman. Graphs, networks and algorithms. Translation from English by MV Gorbatova, V.L Torhova and others. Moscow, The World, 1984.

[5] Cristofides N. The theory of graphs. Algorithmic approach. Translating to Russian language. "The World", 1978.

[6] Nishanbayev T., Muradova A., Research of parameters of the manage-ment level of info communication networks with use of the equipment of the software defined networks. International Journal for Research in Applied Science \& Engineering Technology (IJRASET). Volume 4, Issue IX, September 2016.

[7] Nishanbayev T. N. Muradova A., Research of reliability parameters of transport level of the next generation networks. International Journal for Research in Applied Science \&
Engineering Technology (IJRASET). Volume 4, Issue X, October 2016.

[8] Nishanbayev T. N. Optimization model of the formation in a distributed network of a virtual computing resource. Infocommunications: networks, technologies, solutions, 1 (37) 2016.

[9] Nishanbayev T. N. Formation of a virtual computing resource in a heterogeneous environment. In the collection of the works of the 10th international specialized scientific and technical conference "TECHNOLOGIES OF INFORMATION SOCIETY". Moscow, March 2016.

[10] Nishanbayev T. N., Abdullaev M.M. Problems of the distributed systems in infocommunication media network with complex structure. ITPA 2015 Tashkent 2015.

[11] Nishanbayev T. N. N. Fundamentals of the formation of a service-oriented distributed system based on a programconfigurable network. In the tr. Scientific and Technical Conference of the Republic of Uzbekistan "Creating software in the implementation of modern information and communication technologies: problems and solutions", Samarkand. September 2016.

[12] Nishanbayev T. N. Formalization of the problem of optimal design of next-generation networks. Infocommunications: networks, technologies, solutions, №1 (29) 2014. 